\title{
Progress in halide-perovskite nanocrystals with near-unity- photoluminescence quantum yield
}

\author{
Andrés F. Gualdrón-Reyes ${ }^{1}$, Sofia Masi ${ }^{1}$, and Iván Mora-Seró ${ }^{1, * @ @ ~}$ \\ ${ }^{1}$ Institute of Advanced Materials (INAM), Universitat Jaume I (UJI), Avenida de Vicent Sos \\ Baynat, s/n, 12071 Castellón de la Plana, Spain. \\ *Correspondence: sero@uji.es (I. Mora-Seró) @IvanMoraSero
}

\begin{abstract}
Colloidal halide perovskite nanocrystals (PNCs) are an outstanding case study due to their remarkable optical features, such as a high photoluminescence quantum yield (PLQY), tunable band gap, and a narrow emission. Despite the impressive first reports of PLQY beyond 70\% for PNCs, it has been observed that PLQY is limited by a defective structure, due to the labile interaction between the organic capping ligand and inorganic core. In this line, structural defects acting as trap states are the key factor to limit not just PNCs PLQYs but also their stability. In this review, we present the most studied, common, and alternative protocols to fully compensate for surface defects, mainly halide vacancies and the loss of protective capping ligands, as well as how increase their stability and PLQY up to100\%.
\end{abstract}

Keywords: perovskite nanocrystals; photoluminescence quantum yield; synthetic protocols; ligand passivation; defect suppression; high-quality materials

\section{Defective perovskite nanocrystals after synthesis}

Halide perovskite nanocrystals (PNCs) with $\mathrm{APbX}_{3}$ formulation $\left(\mathrm{A}=\mathrm{Cs}^{+}, \mathrm{FA}^{+}\right.$; formamidinium, $\mathrm{MA}^{+}$; methylamonium, $\mathrm{X}=\mathrm{Cl}, \mathrm{Br}$, I and corresponding mixtures) have received growing interest in optoelectronics, photovoltaics, and analogous solar-driven processes as photocatalysis, extending their applicability during recent years. The outstanding photophysical properties of PNCs such as tunable band gaps covering a broad UV-Vis-IR region [1, 2], narrow full width at half maximum (FWHM) photoluminescence (PL) [3], stability in room ambient conditions [4,5], versatile surface chemistry and facile processability [6-8], have triggered diverse 
studies to prepare PNC colloidal solutions for efficient multicolor light-emitting diodes (LEDs) [9-13], perovskite quantum dot solar cells (QDSSCs) [14-18], and conduct oxidation/reduction reactions on model systems [2, 19, 20], solar fuel generation [21, 22], and solar synthesis [23]. Since the first report provided by Perez Prieto and co-workers [24] and Kovalenko and coworkers[1] on the synthesis of $\mathrm{CH}_{3} \mathrm{NH}_{3} \mathrm{PbBr}_{3}$ and $\mathrm{CsPbX}$ PNCs in 2014 and 2015, respectively, research in the field of PNCs has demonstrated that improvement of the above properties is defined by the quality of the synthesized PNCs, established by estimating their photoluminescence quantum yield (PLQY) [5]. After six years of continuous studies on the intrinsic properties of PNCs, it has been deduced that these materials are denoted as "high-quality" when their PLQY is $>90 \%[5,25,26]$. This means that the radiative recombination mechanism requires the material to be as trap-free as possible and mostly dictates the optoelectronic features of PNCs [27].

In this way, PNCs are commonly preferred as promising light-emitters with high color quality over analogous systems such as 3D perovskite bulk films, where a lower PLQY is usually reported [28]. Although, computational studies have demonstrated that the different defects density in bulk perovskite and PNCs is not enough to explain the difference in the PLQY between them, other main aspects such as the interaction with ligands and solvent, and the location of the defects lead to the control of the surface passivation and in turn of the PLQY, are more easily maximized in the PNCs [29]. Indeed, bulk films also suffer of ion migration promoted in the grain boundaries, favoring the non-radiative recombination dynamics mediated by defects and low-stability [30]. However, the presence of surface-passivating organic ligands, and the spatial confinement of PNCs reduce the emergence of ion migration-mediated defects, improving their PL properties [31]. Interestingly, PNCs colloidal solutions have been used as an additive during bulk film processing [32,33], generating a reduction of defects in bulk by passivating carrier traps through ionic species provided by PNCs and surface passivation by ligand anchoring [34].

Therefore, the fact that high-quality PNCs, with high PLQY, show a low density of intra band gap carrier traps, reducing the non-radiative recombination pathway into PNCs layers, favoring that LEDs reach higher external quantum efficiencies (EQE) greater than $20 \%[13,35,36]$. The reduction of defects has also hindered the loss of carriers during their transportation into high efficient QDSSCs with photoconversion efficiencies of 16.6\% [18], and induce better electron extraction and enhanced photocurrent in photodetectors, with high photoresponsivity/detectivity of $1.4 \times 10^{8} \mathrm{AW}^{-1} / 4.72 \times 10^{15}$ Jones at an incident light $430 \mathrm{~nm}$ [37]. However, despite the impressive PLQYs of the first reports, the fact that the PLQYs of Cs-, FA-PbX ${ }_{3}$ PNCs does not reach unity in many cases [38-41] is clear evidence that these materials are not fully defect-free, and in certain cases, the non-radiative channel is also open for deteriorating their optical properties and long-term stability. 
In this context, diverse research groups agree that the quality of PNCs depends mainly on the synthetic protocol used for material growth. The stoichiometric mixture of $\mathrm{Cs}_{2} \mathrm{CO}_{3}, \mathrm{PbX}_{2}$, and long-chain capping ligands as oleic acid (OA) and oleylamine (OLA) during PNC synthesis, produces a degree of structural defects in the final product. Halide vacancies are the most wellrecognized defects in the PNCs, and they are represented by the emergence of metallic lead, $\mathrm{Pb}^{0}$ [42]. These undercoordinated $\mathrm{Pb}$ species are located in the PNC surface acting as non-radiative recombination centers for electron trapping [43, 44]. The generation of these defects has been also associated with difficulties in stabilizing the PNCs. During PNC preparation, OA and OLA are transformed into oleylammonium oleate, which is the main species for PNC stabilization [5, $45,46]$. Oleylammonium cations bind to halide anions of the PNCs as ionic pairs, promoting surface passivation. Unfortunately, a fraction of the ammonium ligand, now denoted as oleylammonium halide, can be easily detached from the PNC surface, giving place to halide defects $[45,47]$.

Through density functional theory calculations, Chen and co-workers[48] reported that the adsorption energy of the OLA-capped $\mathrm{CsPbI}_{3}(4.599 \mathrm{eV})$ is lower than that of shorter-capping ligands covering the PNC surface as octylamine (OctA, $5.022 \mathrm{eV}$ ). In this case, the OctA (Lewis base ligand) preferentially coordinates with metal atoms of the surface. OctA-CsPbI ${ }_{3} \mathrm{PNCs}$ exhibited an increase in long-term stability for 6 months, with a PLQY 94\%, reducing iodide defects and retaining the cubic phase, as stabilization of perovskite black phase is an additional advantage of the PNC synthesis [49]. Thus, the dynamic ligand binding nature is the initial step towards the stabilization and depends on the type of ligands, as well as on the PNC surface.

Clearly, additional strategies supporting the most common use of ammonium ligands, that maximize the radiative recombination pathway, along with the passivation of halide deficiency, are desirable to reach a $100 \%$ PLQY. In this review, we highlight the main approaches to achieve PNCs with 100\% PLQY, based on the purification stage, surface post-synthetic treatments, partial $\mathrm{Pb}$ substitution/cation doping and modified synthetic routes to produce high-quality materials for perovskite-based technologies.

\section{Purification of the perovskite nanocrystals}

One of the main problems associated with PNCs is maintaining or improving of their optical and structural properties during isolation and purification steps following synthesis. Variations in the purification stage have been mostly considered in order to evaluate the quality of the final product [50-52]. On one hand, the addition of antisolvents to the crude reaction has been reported to decrease the PLQY of PNCs (from $70 \%$ to $30 \%$ for $\mathrm{CsPbBr}_{3}$ and from $80 \%$ to $0 \%$ for $\mathrm{CsPbI}_{3}$ ) caused by the detachment of a high amount of capping ligands from the PNC surface [48, 53, 54]. This leads to several issues such as PNC agglomeration, a wide particle size distribution (broad 
PL FWHM) and thereby, the preference for the non-photoactive yellow $\boldsymbol{\delta}$-phase in the case of iodide perovskites $[49,51,52]$. However, the PNCs isolation in absence of antisolvents causes a high density of nanoparticles (NPs) to be dispersed in the supernatant, which is then discarded, reducing the product yield [51]. Therefore, the purification process to achieve a high amount of PNCs without altering their optical properties is still under study.

Experimental parameters such as the antisolvent nature, solvent:antisolvent volume ratio, and centrifuge rate and time also influence PNCs isolation. Zhang and co-workers [51] studied the compatibility of some $\mathrm{PNCs}$ such as $\mathrm{CsPbBr}_{3}, \mathrm{CsPbI}_{3}$, and some antisolvents with different polarity, as the case of methanol, 1-butanol, acetonitrile, acetone, methyl acetate (MeOAc), and ethyl acetate (EtOAc) (Figure 1a). Here, a centrifugation rate of $8000 \mathrm{rpm}$ for $5 \mathrm{~min}$ and a 1:1 volume ratio of solvent:antisolvent were established. Then, PNCs were redispersed in hexane and centrifugate again at $8500 \mathrm{rpm}$ for $5 \mathrm{~min}$. At this point, the supernatant was recovered as a purified product. By measuring the PL spectra of purified PNCs (Figure 1b), and by comparing their optical features with a luminescent standard system like Rhodamine $\mathrm{B}$, it was found that $\mathrm{CsPbr}_{3}$ was stable in all the antisolvents, reaching a PLQY $>90 \%$ by washing with methanol and unityPLQY after washing with acetonitrile. In the case of $\mathrm{CsPbI}_{3}$ PNCs, highly polar antisolvents decrease the PLQY considerably, promoting the emergence of a yellow $\delta$-phase and the loss of the optical properties.

Although the PLQY of PNCs using MeOAc and EtOAc is $<70 \%$ (Figure 1b), these antisolvents are commonly chosen for a higher PNC product yield to facilitate their applications, especially in LEDs [55, 56]. On the other hand, Konstantatos and co-workers[57] proposed a facile route to enhance the photophysical properties of $\mathrm{FAPbBr}_{3} \mathrm{PNCs}$ synthesized with shortchain capping ligands as octylamine and octanoic acid: the simultaneous addition of OA and $\mathrm{PbBr}_{2}$ to the redispersed material in toluene, prior to purification with $\mathrm{MeOAc}$. The presence of $\mathrm{OA}$ removes the presence of $\mathrm{PNC}$ aggregates, while $\mathrm{PbBr}_{2}$ provides an excess of $\mathrm{Br}^{-}$anions to passivate the halide defects and strengthen the ligand binding in the PNC surface $[57,58]$. This procedure affords purified $\mathrm{FAPbBr}_{3}$ PNCs with a PL peak emission at $511 \mathrm{~nm}, \mathrm{FWHM}=22 \mathrm{~nm}$, and PLQY around $90 \pm 9 \%$ (Figure 1c). The improvement of PLQY was accompanied with a change in the PL dynamics of the PNCs, where the carrier lifetime was longer for purified materials compared with the as-synthesized ones (Figure 1d). This fact is associated to the reduction of the non-radiative recombination paths, providing efficient photoluminescence. 


\section{Surface restoration of the perovskite nanocrystals}

\section{Stronger ligand binding to perovskite core}

One of the most recognized strategies to compensate for the loss of organic ligands from the shell life of the $\mathrm{CsPbr}_{3} \mathrm{PNCs}$ is through post-synthetic treatment with quaternary ammonium salts, namely didodecyldimethylammonium bromide (DDAB) $[53,59,60]$. Compared with the linked OLA that shows a high likelihood of desorption, $\mathrm{DDA}^{+}$cations produce stronger binding with the negatively charge surface sites of the perovskite $[61,62]$. This induces the formation of hydrophobic monolayers, increasing the long-term stability of the PNCs. The effect of DDAB can be also combined with the addition of $\mathrm{PbBr}_{2}$, used for repairing the $\mathrm{PbBr}_{6}$ octahedra from the $\mathrm{Pb}$ - or/and Br-deficiency. Bodnarchuk and co-workers [53] have incorporated both DDAB and $\mathrm{DDAB}+\mathrm{PbBr}_{2}$ to $\mathrm{CsPbBr}$ colloidal solutions, which showed a low PLQY near to $60-70 \%$ before purification and $30-40 \%$ after purification, respectively. After post-synthetic treatment, the PLQY of the samples raised to $100 \%$. As shown in Figure 2a, the visual brightness of the samples in the presence of $\mathrm{DDAB}$ or $\mathrm{DDAB}+\mathrm{PbBr}_{2}$ is increased, reflecting the enhancement of the optical features, and indicating that electron relaxation is performed radiatively. The surface modification does not have a marked impact on the PL peak position of the $\mathrm{CsPbBr}_{3} \mathrm{PNCs}$, exhibiting an emission peak between 512-514 nm, with a PL FWHM between 18-20 nm (Figure 2b). In this line, the use of three-ligand surface engineering where octanoic acid is pivotal for improving the optoelectronic features of $\mathrm{CsPbrr}_{3} \mathrm{PNCs}_{\text {[63] }}$, and the addition of zwitterionic ligands with strong binding to PNCs surface $[64,65]$ are also considered promising strategies to achieve an efficient defect passivation of PNCs surface.

Therefore, the addition of efficient capping ligands during synthesis has been considered to provide better protection of the PNC surface [66-69]. Shen and co-workers [70] introduced trioctylphosphine (TOP) as a capping agent to be mixed with $\mathrm{PbI}_{2}$, in order of achieving PNCs with a more perfect structure and suppressed surface defects. In this case, lone-pair electrons from $\mathrm{P}$ atoms are donated to Lewis acid $\mathrm{Pb}^{2+}$ cations, forming a TOP. $\mathrm{PbI}_{2}$ adduct to facilitate the $\mathrm{PbI}_{2}$ dissolution and generate more efficient passivation. Accordingly, TOP-CsPbI ${ }_{3} \mathrm{PNCs}$ show narrow PL FWHM $\sim 30 \mathrm{~nm}$, reduced carrier trap density, and thereby, a 100\% PLQY compared with the TOP-free material, exhibiting values between $78-84 \%$. The suitable passivation of surface defects by TOP allows for control of the PNC size by varying the synthesis temperature (particle size), obtaining a stable unity-PLQY (Figure 2c). Simultaneously, the high-quality of TOP-CsPbI ${ }_{3}$ PNCs is visible after washing with $\mathrm{MeOAc}$ and storing for one month, with a decrease of only around $15 \%$ of the initial PLQY value. In contrast, standard $\mathrm{CsPbI}_{3} \mathrm{PNCs}_{\text {with }}$ lower initial PLQY display a marked decrease around 26\% (Figure 2d), indicating of a poorer passivated surface in the time. In addition to the enhancement of PLQY, PNCs also shows a 
change in its PL decay behavior after carrying out an efficient surface passivation (Figure 2e). Unlike defective PNCs with low PLQY, exhibiting PL decay with multiexponential character, PNCs with near-unity PLQY display transient PL kinetics close to single-exponential character. This fact indicates that non-radiative recombination sites are suppressed to maximize the radiative channel $[68,71]$.

Removal of unloaded defect sites - fixing the surface stoichiometry.

Alivisatos and co-workers [71] have demonstrated the enhancement of PL properties of $\mathrm{Cs}_{\mathrm{PbBr}} \mathrm{PNCs}_{3}$ by using thiocyanate salts such as $\mathrm{NH}_{4} \mathrm{SCN}$ or $\mathrm{NaSCN}$, to repair a lead-rich PNC surface and extending the carrier lifetime into the material (Figure $2 \mathrm{e}$ ). $\mathrm{SCN}^{-}$species are able to remove excess $\mathrm{Pb}^{0}$ formed during the synthesis, forming $\mathrm{Pb}-\mathrm{S}$ bonds. This provides a suitable surface stoichiometry $(\mathrm{Pb}: \mathrm{Br}=1: 3)$ without causing structural changes in the nanoparticles. The removal of $\mathrm{Pb}^{0}$ decreases the density of carrier traps in the PNCs [72], trendering the radiative PL decay more efficient. By comparing the optical properties of fresh and aged $\mathrm{CsPbBr}_{3} \mathrm{PNCs}$ samples before and after $\mathrm{SCN}^{-}$treatment, PLQY was maximized: from $92 \pm 2 \%$ to $99 \pm 2 \%$ for the case of as-prepared PNCs, while aged ones showed an improvement from $63 \pm 2 \%$ to $100 \pm$ $3 \%$. Ahmed and co-workers [44] have added $\mathrm{NaBF}_{4} / \mathrm{NH}_{4} \mathrm{BF}_{4}$ to $\mathrm{CsPbBr} 2 \mathrm{Cl}$ colloidal solutions, boosting the PLQY from $4 \pm 1 \%$ to $96 \pm 3 \%$. Here, $\mathrm{Pb}^{0}$ species are removed by using $\mathrm{BF}_{4}{ }^{-}$anions. On the other hand, $\mathrm{CsPbI}_{3}$ PNCs with 100\% PLQY has not been reached in the presence of salts (e.g., $\mathrm{SCN}^{-}$or $\mathrm{ZnI}_{2}$ ) [26, 72]. This is attributed to the high iodide lability, generating halide vacancies and a fast phase transformation ( $\alpha$ - to $\delta$-phase) [49]. Consequently, the effectiveness of the salt treatment decreases.

\section{Partial Pb substitution - doping}

The addition of metal halides, including metal doping during or after PNC synthesis has been studied with the goal of (i) tuning/enhancing the photophysical properties of the materials, and (ii) decreasing the fraction of $\mathrm{Pb}$ to push commercialization of perovskite-based technologies $[25$, $73,74]$. However, some metals can introduce new non-radiative paths for carrier recombination, quenching the PL of the host [75], while in other cases, no modifications in the optical features are observed just as in the absence of the metal substitute $[76,77]$. However, some transition, rare-earth metals or lanthanides have been studied deeply to improve the optoelectronic properties of PNCs with better phase stability through dual metal-doping [78-80].

Ji and colleagues [81] have shown enhancement of PL properties of $\mathrm{Mn}^{2+}$-doped $\mathrm{CsPCl}_{3}$ PNCs by adding $\mathrm{CdCl}_{2}(100 \mu \mathrm{L}$ of $33 \mathrm{mM} \mathrm{CdCl})$ in ethanol as a post-synthetic treatment. Attending to the the PLQY of standard $\mathrm{CsPCl}_{3}$ is low $(<10 \%)[77,82], \mathrm{Mn}^{2+}$ energy levels are 
introduced in the band gap of the PNCs, causing orange-red light emission and making more efficient the radiative carrier recombination dynamics. However, a maximum PLQY around 62\% was achieved for the $\mathrm{Mn}: \mathrm{Pb}$ molar ratio 3:1, due to the presence of high density of non-radiative recombination sites formed by atomic vacancies into $\mathrm{CsPCl}_{3}$ host. These species block the $\mathrm{Mn}^{2+}$ $d-d$ transition, generating octahedra tilting of $\mathrm{PbCl}_{6}$ units $[77,83]$. Through PNC dual-surface passivation with $\mathrm{Cd}^{2+}$ and $\mathrm{Cl}^{-}$vacancies are rapidly filled, reducing the octahedra distortion and providing a unity-PLQY (Figure 3a). This is accompanied with the lengthening of the PL lifetime, corroborating the reduction of non-radiative sites from the PNCs. Simultaneously, $\mathrm{CdCl}_{2}-\mathrm{Mn}^{2+}-$ $\mathrm{CsPCl}_{3} \mathrm{PNCs}$ are not affected by subsequent purifications (Figure $3 \mathrm{~b}$ ), only reducing $\sim 11 \%$ from the initial PLQY. Because the ionic radius of $\mathrm{Cd}^{2+}(0.95 \AA)$ is smaller than that of $\mathrm{Pb}^{2+}(1.19 \AA)$, the Goldschmidt tolerance factor is increased, producing a less defective structure with higher phase stability $[77,84]$.

Furthermore, Gamelin and co-workers[85] have introduced $\mathrm{Yb}^{3+}$ cations into $\mathrm{CsPbCl}_{3} \mathrm{PNCs}$ (doping 5.2\%), raising the PLQY above the $100 \%$ in the near-infrared (NIR) region. Taking advantage from ${ }^{2} \mathrm{~F}_{5 / 2} \rightarrow{ }^{2} \mathrm{~F}_{7 / 2} f-f$ emission of $\mathrm{Yb}^{3+}$, which is an effective activator of quantum cutting (one high-energy photon is absorbed and then two-low energy photons are generated) [78, 86], the carrier transfer from the host is accelerated, improving the radiative relaxation of the modified $\mathrm{CsPbl}_{3}$. After $\mathrm{Yb}^{3+}$-doping and varying the intensity of the excitation source $(\lambda=380$ $\mathrm{nm}$ ), the highest PLQY around 170\% was achieved (Figure 3c). This value is the highest reported for these materials and closer to the theoretical $200 \%$ for the visible-to-NIR quantum cutting in presence of $\mathrm{Yb}^{3+}$.

It has been demonstrated that the partial replacement of $\mathrm{Pb}$ by $\mathrm{Sr}$ during synthesis of $\mathrm{FAPb}_{1-\mathrm{x}} \mathrm{Sr}_{\mathrm{x}} \mathrm{I}_{3} \mathrm{PNCs}$ [87] or $\mathrm{CsPbI}_{3}[25]$ generates red-emitting materials with high-quality. As $\mathrm{Sr}^{2+}$ cations exhibit the same ionic radius as $\mathrm{Pb}^{2+}[25]$, it is expected that $\mathrm{SrI}_{2}$ can easily substitute $\mathrm{PbI}_{2}$ in the mixture reaction, producing less-toxic PNCs, keeping their intrinsic properties. After

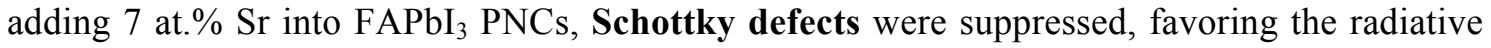
recombination pathway. Thus, the PLQY of pristine $\mathrm{FAPbI}_{3}$ was maximized from $93 \%$ to $100 \%$, showing a blue shift in the PL features (Figure 3d). Interestingly, the excess Sr content generates the alteration of stoichiometry of the precursors during the PNCs synthesis, opening the door to distinct morphologies with different PL properties. In addition, $\mathrm{Sr}$ doping also increases significantly the stability, preserving the $80 \%$ of the initial PLQY after 6.5 months.

\section{Modified synthetic routes for synthesizing high-quality perovskite nanocrystals}

One encouraging approach to prepare high-quality PNCs is through the ligand-assisted reprecipitation technique (LARP). This protocol is simpler, lower- cost, faster, and requires milder conditions, being suitable for organic nanomaterials [88, 89]. Pérez-Prieto and co- 
workers[90] have synthesized $\mathrm{MAPbBr}_{3}$ nanoplatelets with unity-PLQY, by preparing solutions containing certain amount of $\mathrm{MABr}, \mathrm{PbBr}_{2}$ and 2-adamantylammonium bromide (ADBr) in DMF. A highly green-luminescent material is obtained after adding the mixture in a poor solvent such as toluene under stirring. Considering that $\mathrm{MAPbBr}_{3}$ PNCs shows a PLQY near to 93\% when OctA is used, the incorporation of $\mathrm{ADBr}$ provides better coverage of the material surface, reaching an effective passivation and improving the PL properties. A recent strategy to achieve $\mathrm{MAPbBr}_{3}$ PNCs with $100 \%$ PLQY is by adding a solution of $\mathrm{MABr} / \mathrm{PbBr}_{2} / \mathrm{OctA}$ in DMF in toluene by spray pyrolysis. Lin and co-workers [91] explain that this methodology guarantees a larger contact surface area and better mixing between the good and poor solvent, improving the crystallization of the material.

Meanwhile, Sum and co-workers[92] have repaired the surface defects of $\mathrm{MAPbBr}_{3} \mathrm{PNCs}$ in presence of benzyl alcohol $(\mathrm{BnOH})$, through LARP. Here, $\mathrm{BnOH}$ is able to mediate the ligand coverage and influence the ligand binding motifs, unlocking unity- PLQY. After analyzing highresolution XPS O 1s spectra of the as-prepared $\mathrm{MAPbBr}_{3}$ samples with (Figure 4a) and without (Figure $4 \mathrm{~b}$ ) of $\mathrm{BnOH}$, two different chemical environments of the carboxylate oxygen atoms from OA linked to the PNC surface were observed; region I associated to the bidentate chelating binding mode, while region III is ascribed to monodentate chelating binding mode (Figure 4c). Region II was attributed to O-H species absorbed during sample preparation. It was concluded that the bidentate mode promotes a stronger binding to the $\mathrm{Pb}^{2+}$ cations from the PNCs surface, reducing the density of carrier traps.

Interestingly, Kovalenko and co-workers [93] show a modification of the LARP protocol to prepare $\mathrm{MAPbBr}_{3} \mathrm{PNCs}$, by introducing the amino acids (AAs) as L-lysine and L-arginine in presence of hexanoic acid. For this purpose, the $\alpha$-amino group from both AAs were blocked with a protecting group based on tertbutyloxycarbonyl (tBoc), which (i) forces the selective bonding between the positively amine-groups from AAs and the PNCs core to produce an efficient passivation, and (ii) the blocked functional groups are then free to react with precursors in the mixture reaction to provide a more effective PNCs growth. In this context, tBoc-AAs offer the formation of highly blue-emissive PNCs (Figure 4d) with particle sizes $<6 \mathrm{~nm}$, low size distribution and a PLQY up to $94 \pm 5 \%$ (Figure 4e).

Other alternatives are based on synthetic routes using three precursors (each one for $\mathrm{Cs}, \mathrm{Pb}$ and $\mathrm{Br}$, respectively), where several bromide sources (such as $\mathrm{TOP}-\mathrm{Br}_{2}, \mathrm{~N}$-bromosuccinimide, trimethylsilyl bromide, benzoyl bromide, alkyl-ammonium bromides, ionic liquids, among others) $[64,94-99]$ are added independently from the lead precursor (lead acetate or lead oxide) for $\mathrm{CsPBr}_{3}$ synthesis. This method aims to reduce the stoichiometric excess of $\mathrm{Pb}$ used in the synthesis and source of halide vacancies, which activate non-radiative recombination pathways, as discussed above. However, its main limitation regards the achievement of highly emissive nanoparticles with a shape different from the classical nanocubes (i.e., nanorods). Improved 
methods reported by Grisorio and co-workers[100] were investigated for controlling the shape and stability of $\mathrm{CsPbBr}_{3}$ PNCs while maintaining their high emission efficiency, mainly by controlling the reaction kinetics.

Regarding to the hot-injection method, some modifications have been also performed to improve the quality of the PNCs. It has been demonstrated that the increase of OA/OLA concentration in the mixture reaction facilitates both the stabilization of lead halide during the synthesis and the PNCs growth at temperatures between $170-190{ }^{\circ} \mathrm{C}$ [5]. Meanwhile, Pradhan and co-workers [97] have described a generic synthetic approach to inject oleylammonium halide salts into an amine/halide-free mixture reaction containing an equimolar ratio of $\mathrm{Pb}$ and $\mathrm{Cs}$ at high temperatures $\left(220-260^{\circ} \mathrm{C}\right)$. Both modified routes ensure an efficient ligand surface passivation of PNCs with near-unity PLQY.

Attending to the overall strategies shown along the manuscript (summarized in Figure 5a), we observe that they have been widely studied in Br-PNCs and I-PNCs systems. However, the high halide deficiency generated by the dynamic binding of iodide anions in the PNCs surface [2] makes that some approaches lose effectiveness. In this sense, the nature of the halide affects the strategy in order to boost PLQY of PNCs to near to one values. Thus, new synthetic routes and partial $\mathrm{Pb}$ replacement are suitable for reaching I-PNCs with high quality. On the contrary, the strong complexion affinity of the $\mathrm{Pb}-\mathrm{Br}$ bond makes that $\mathrm{Br}$-PNCs show a more tolerant-to-defect structure. This fact allows us to understand why the abovementioned strategies (mainly ligand passivation and salt treatments) are more effective in Br-PNCs to suppress their surface defects and reach 100\% PLQY more easily. Lastly, the inclusion of metal chloride salts and doping are established as the most reported approaches to promote defect passivation of Cl-PNCs. By considering that $\mathrm{Cl}$-vacancies are associated to intra band gap deep states compared with $\mathrm{Br}$ - and I-vacancies (shallow states) [68], we believe that the reduction of halide deficiency by adding external Cl-species is the most efficient way to produce high-quality blue-emitting Cl-PNCs. In this way, Figure $5 \mathrm{~b}$ shows the developing of the different strategies reported along the time to obtain less-defective PNCs with improved PLQY up to 100\%. For this figure we have considered only reported results of PLQY after PNCs washing/purification and not from raw PNC just after synthesis, as the washed PNCs are the ones susceptible to be incorporated in optoelectronic devices.

\section{Concluding remarks and future perspectives}

In this review, we discussed the main strategies shown in the current state-of-the-art for obtaining high-quality PNCs, with improved photophysical properties, and 100\% PLQY. These strategies point out that the challenge to prepare PNCs with high optical performance, resides on a suitable repair or ligand coverage of the material surface, in order to passivate the surface defects 
and reduce the non-radiative recombination centers. In this context, an efficient ligand coverage and a suitable surface stoichiometry can be reached by using adequate antisolvents during the purification stage and performing the surface restoration by using promising capping agents or salts with high capability to remove defect centers. Meanwhile, the partial $\mathrm{Pb}$ substitution or the use of promising protocols for PNCs synthesis are considered pivotal to suppress surface defects and favor the radiative recombination dynamics. From our point of view, strategies based on ligand passivation and the suppression of halide defects by incorporation of metal halide salts/partial $\mathrm{Pb}$ substitution are the most effective ways to ensure the formation of less-defective PNCs. This is because PNCs are prone to suffer the detachment of alkylammonium halide species during aging, generating a high density of halide defects to damage the material surface. In the case of iodide perovskites, the emergence of high halide deficiency causes the transformation of photoactive $\alpha$-black phase to inactive yellow $\delta$-phase, losing completely their optical properties and stability. Stable PNCs with near-unity PLQY can be achieved by filling the halide vacancies and favoring an adequate ligand coverage by (i) increasing the ligand content or (ii) using stronger binding capping agents. In this general context halide nature has to be taken into account in order to refine PLQY optimization.

We also address future opportunities (see Outstanding Questions) to consider in the development of novel protocols for PNCs preparation such as synthesis parameters, the organic, inorganic or hybrid nature of the material, and the substitution of conventional ligands by potential candidates to mediate PNCs growth with remarkable PL features. At this point, it is essential to understand how the functional groups from promising capping agents can offer a stronger binding to the PNCs core without requiring additional post-treatments. Future studies are needed for conducting the synthesis of unity-PLQY lead-free PNCs by supporting the total $\mathrm{Pb}$ replacement with substitutes that guarantee a less defective material. Moreover, the choice of hydrophobic ligand could be optimized not only for the successfully passivation of the NCs surface, but also to increase the stability in water and the relative application. These definitively are the big goals to boost the efficiencies and the commercialization of perovskite-based technologies.

\section{Conflicts of interest}

The authors declare no competing interests.

\section{Acknowledgements}

Financial support from the European Research Council (ERC) via Consolidator Grant (724424 No-LIMIT), Ministry of Science and Innovation of Spain under Project STABLE PID2019- 
107314RB-I00 and Generalitat Valenciana via Prometeo Grant Q-Devices (Prometeo/2018/ 098) is gratefully acknowledged.

\section{Glossary}

Capping ligands: also known as stabilizing agents, are organic molecules, surfactants or polymers employed to coat nanoparticles. These agents show an amphiphilic structure, where the polar head coordinates with the particle core, while the non-polar tail interact with the external medium. Capping ligands are useful for hindering the aggregation and controlling the material size and shape, and also to passive surface defects.

Carrier lifetime: is referred as the average time that an excess of minor carriers takes to recombine. This process can be performed via band-to-band, trap-to-band and Auger recombination pathways.

Full width at half maximum: is defined to the width of a distribution curve measured between two points on the $y$-axis which are in the half of the maximum value.

Halide perovskite nanocrystals (PNCs): are a class of semiconductors (with size $\sim 2-20 \mathrm{~nm}$ large) with an $\mathrm{ABX}_{3}$ type structure, where in most of the studies $\mathrm{A}=\mathrm{Cs}^{+}, \mathrm{MA}^{+}, \mathrm{FA}^{+} ; \mathrm{B}=\mathrm{Pb}^{2+}$, $\mathrm{Sn}^{2+}$ and $\mathrm{X}^{-} \mathrm{Cl}^{-}, \mathrm{Br}^{-}, \mathrm{I}^{-}$, or combinations, exhibiting unique features different to other conventional nanosized materials or quantum dots. These outstanding characteristics are based mainly on a higher absorption coefficient, longer diffusion length, higher carrier mobility, tolerant-to-defect structure, and tunable optical properties by controlling quantum-size effects. To highlight, some of these PNCs can emit brightly without performing ligand passivation, and they can be good bright emitters even under larger particle size. This fact makes that PNCs showing polydispersity can provide narrow linewidths. By attending these attributes and the low-cost and facile synthesis, the commercial interest to use PNCs has increased in optoelectronic applications such as lightemitting diodes, photodetectors, solar cells, among others.

Non-radiative recombination: is referred to the trap-to-band transitions, where the electrons in the $\mathrm{CB}$ are trapped in new energy states formed into the band gap of the material by a dopant or a structural defect. The generated energy is released as a lattice vibration, forming a phonon instead a photon. Unlike radiative recombination, this process reduces the PLQY and increases the thermal energy into the material. 
Perovskite black phase: is referred as the photoactive polymorph of iodide perovskites, formed by the dynamic motion of $\left[\mathrm{PbI}_{6}\right]$ octahedra to form a highly symmetric crystal structure. Depending on the distortion of octahedra, different optically phases such as $\alpha$-, $\beta$ - and $\gamma$-phases are obtained.

Photoluminescence quantum yield (PLQY): is an optical property measuring the number of photons emitted in fraction to the number of photons adsorbed. PLQY can be calculated in a range between $0-100 \%$, being unity the indication that a high-quality material, with reduced density of defects, can emit the same number of collected photons.

Radiative recombination: is referred to the band-to-band transitions, where the electrons in a high energy level (conduction band, CB) decay to a lower energy empty level (valence band, VB), emitting the generated energy in form of photon.

Schottky defects: In ionic solids, are referred to the structural imperfections generated by atomic vacant positions. These vacancies are created by the movement of opposite ions from the interior to the material surface, leaving opposite charged vacancies. Schottky defects are produced stoichiometrically to preserve the electroneutrality in the solid.

Surface passivation: is referred to the formation of a protective shell layer on the material surface through a chemical reaction, which support the reduction of surface defects, improves, and preserves the intrinsic properties of the material. In this context, a passivated material is less affected by the external environment.

Yellow $\delta$-phase: is referred to the non-photoactive polymorph of iodide presenting a nonperovskite crystalline structure formed by the destabilization of the perovskite structure at room temperature. This phase shows a non-perovskite structure with double-chains of non-cornersharing $\left[\mathrm{PbI}_{6}\right]$ octahedra.

\section{References}

1. Protesescu, L. et al. (2015) Nanocrystals of Cesium Lead Halide Perovskites (CsPbX3, X $=\mathrm{Cl}$, $\mathrm{Br}$, and I): Novel Optoelectronic Materials Showing Bright Emission with Wide Color Gamut. Nano Letters 15 (6), 3692-3696

2. Gualdrón-Reyes, A. F. et al. (2020) Unravelling the Photocatalytic Behavior of All-Inorganic Mixed Halide Perovskites: The Role of Surface Chemical States. ACS Applied Materials \& Interfaces 12 (1), 914-924 
3. Du, X. et al. (2017) High-quality $\mathrm{CsPbBr3}$ perovskite nanocrystals for quantum dot lightemitting diodes. RSC Advances 7 (17), 10391-10396

4. Liu, F. et al. (2017) Colloidal Synthesis of Air-Stable Alloyed CsSn1-xPbxl3 Perovskite Nanocrystals for Use in Solar Cells. Journal of the American Chemical Society 139 (46), 1670816719

5. Hassanabadi, E. et al. (2020) Ligand \& Band Gap Engineering: Tailoring the Protocol Synthesis for Achieving High-Quality CsPbl3 Quantum Dots. Nanoscale 12, 14194-14203

6. Grisorio, R. et al. (2019) Exploring the surface chemistry of cesium lead halide perovskite nanocrystals. Nanoscale 11 (3), 986-999

7. Nedelcu, G. et al. (2015) Fast Anion-Exchange in Highly Luminescent Nanocrystals of Cesium Lead Halide Perovskites (CsPbX3, X $=\mathrm{Cl}, \mathrm{Br}$, I). Nano Letters 15 (8), 5635-5640

8. Park, D. H. et al. (2018) Facile synthesis of thermally stable CsPbBr3 perovskite quantum dotinorganic $\mathrm{SiO} 2$ composites and their application to white light-emitting diodes with wide color gamut. Dyes and Pigments 149, 246-252

9. Lu, M. et al. (2018) Highly Flexible CsPbl3 Perovskite Nanocrystal Light-Emitting Diodes. ChemNanoMat 5 (3), 313-317

10. Shen, X. et al. (2019) Zn-Alloyed CsPbl3 Nanocrystals for Highly Efficient Perovskite LightEmitting Devices. Nano Letters 19 (3), 1552-1559

11. Zhang, C. et al. (2020) Core/Shell Perovskite Nanocrystals: Synthesis of Highly Efficient and Environmentally Stable FAPbBr3/CsPbBr3 for LED Applications. Advanced Functional Materials 30 (31), 1910582

12. Chiba, T. et al. (2018) Anion-exchange red perovskite quantum dots with ammonium iodine salts for highly efficient light-emitting devices. Nature Photonics 12 (11), 681-687

13. Lin, K. et al. (2018) Perovskite light-emitting diodes with external quantum efficiency exceeding 20 per cent. Nature 562 (7726), 245-248

14. Li, F. et al. (2019) Perovskite Quantum Dot Solar Cells with $15.6 \%$ Efficiency and Improved Stability Enabled by an $\alpha$-CsPbI3/FAPbI3 Bilayer Structure. ACS Energy Letters 4 (11), 2571-2578 15. Xue, J. et al. (2018) Surface Ligand Management for Stable FAPbI3 Perovskite Quantum Dot Solar Cells. Joule 2 (9), 1866-1878

16. Sanehira, E. M. et al. (2017) Enhanced mobility CsPbl3 quantum dot arrays for recordefficiency, high-voltage photovoltaic cells. Science Advances 3 (10), eaao4204

17. Zhang, L. et al. (2020) All-Inorganic CsPbI3 Quantum Dot Solar Cells with Efficiency over $16 \%$ by Defect Control. Advanced Functional Materials, 2005930

18. Hao, M. et al. (2020) Ligand-assisted cation-exchange engineering for high-efficiency colloidal Cs1-xFAxPbI3 quantum dot solar cells with reduced phase segregation. Nature Energy 5 (1), 79-88

19. Cardenas-Morcoso, D. et al. (2019) Photocatalytic and Photoelectrochemical Degradation of Organic Compounds with All-Inorganic Metal Halide Perovskite Quantum Dots. The Journal of Physical Chemistry Letters 10 (3), 630-636

20. Xu, Y.-F. et al. (2017) A CsPbBr3 Perovskite Quantum Dot/Graphene Oxide Composite for Photocatalytic CO2 Reduction. Journal of the American Chemical Society 139 (16), 5660-5663

21. Pavliuk, M. V. et al. (2018) Hydrogen evolution with CsPbBr3 perovskite nanocrystals under visible light in solution. Materials Today Communications 16, 90-96

22. Poli, l. et al. (2019) Graphite-protected $\mathrm{CsPbBr} 3$ perovskite photoanodes functionalised with water oxidation catalyst for oxygen evolution in water. Nature Communications 10 (1), 2097

23. Chen, K. et al. (2017) Photocatalytic Polymerization of 3,4-Ethylenedioxythiophene over Cesium Lead lodide Perovskite Quantum Dots. Journal of the American Chemical Society 139 (35), 12267-12273

24. Schmidt, L. C. et al. (2014) Nontemplate Synthesis of $\mathrm{CH} 3 \mathrm{NH} 3 \mathrm{PbBr} 3$ Perovskite Nanoparticles. Journal of the American Chemical Society 136 (3), 850-853 
25. Yao, J.-S. et al. (2019) Few-Nanometer-Sized $\alpha$-CsPbI3 Quantum Dots Enabled by Strontium Substitution and lodide Passivation for Efficient Red-Light Emitting Diodes. Journal of the American Chemical Society 141 (5), 2069-2079

26. Li, F. et al. (2018) Postsynthetic Surface Trap Removal of CsPbX3 (X $=\mathrm{Cl}, \mathrm{Br}$, or I) Quantum Dots via a ZnX2/Hexane Solution toward an Enhanced Luminescence Quantum Yield. Chemistry of Materials 30 (23), 8546-8554

27. Yao, J.-S. et al. (2020) Suppressing Auger Recombination in Cesium Lead Bromide Perovskite Nanocrystal Film for Bright Light-Emitting Diodes. The Journal of Physical Chemistry Letters 11 (21), 9371-9378

28. Xing, G. et al. (2017) Transcending the slow bimolecular recombination in lead-halide perovskites for electroluminescence. Nature Communications 8 (1), 14558

29. ten Brinck, S. et al. (2019) Defects in Lead Halide Perovskite Nanocrystals: Analogies and (Many) Differences with the Bulk. ACS Energy Letters 4 (11), 2739-2747

30. Kim, Y.-H. et al. (2018) Charge carrier recombination and ion migration in metal-halide perovskite nanoparticle films for efficient light-emitting diodes. Nano Energy 52, 329-335

31. Kim, Y.-H. et al. (2017) Highly Efficient Light-Emitting Diodes of Colloidal Metal-Halide Perovskite Nanocrystals beyond Quantum Size. ACS Nano 11 (7), 6586-6593

32. Zhang, Y. et al. (2019) Fusing Nanowires into Thin Films: Fabrication of GradedHeterojunction Perovskite Solar Cells with Enhanced Performance. Advanced Energy Materials 9 (22), 1900243

33. Yang, H. et al. (2018) Building bridges between halide perovskite nanocrystals and thin-film solar cells. Sustainable Energy \& Fuels 2 (11), 2381-2397

34. Zheng, X. et al. (2019) Quantum Dots Supply Bulk- and Surface-Passivation Agents for Efficient and Stable Perovskite Solar Cells. Joule 3 (8), 1963-1976

35. Dong, Y. et al. (2020) Bipolar-shell resurfacing for blue LEDs based on strongly confined perovskite quantum dots. Nature Nanotechnology 15 (8), 668-674

36. Kim, Y.-H. et al. (2021) Comprehensive defect suppression in perovskite nanocrystals for high-efficiency light-emitting diodes. Nature Photonics 15 (2), 148-155

37. Pradhan, B. et al. (2020) Ultrasensitive and ultrathin phototransistors and photonic synapses using perovskite quantum dots grown from graphene lattice. Science Advances 6 (7), eaay5225 38. R, S. et al. (2020) Green to Blue Light Emitting CsPbBr3 Perovskite by Ligand Exchange and its Encapsulation by $\mathrm{TiO} 2$ for Tandem Effect in Photovoltaic Applications. ACS Applied Nano Materials 3 (6), 6089-6098

39. Jancik Prochazkova, A. et al. (2020) Controlling Quantum Confinement in Luminescent Perovskite Nanoparticles for Optoelectronic Devices by the Addition of Water. ACS Applied Nano Materials 3 (2), 1242-1249

40. Ahmed, G. H. et al. (2018) Giant Photoluminescence Enhancement in CsPbCl3 Perovskite Nanocrystals by Simultaneous Dual-Surface Passivation. ACS Energy Letters 3 (10), 2301-2307

41. Protesescu, L. et al. (2017) Dismantling the "Red Wall" of Colloidal Perovskites: Highly Luminescent Formamidinium and Formamidinium-Cesium Lead lodide Nanocrystals. ACS Nano 11 (3), 3119-3134

42. Seth, S. et al. (2019) Tackling the Defects, Stability, and Photoluminescence of CsPbX3 Perovskite Nanocrystals. ACS Energy Letters 4 (7), 1610-1618

43. Wang, S. et al. (2018) Cesium Lead Chloride/Bromide Perovskite Quantum Dots with Strong Blue Emission Realized via a Nitrate-Induced Selective Surface Defect Elimination Process. The Journal of Physical Chemistry Letters 10 (1), 90-96

44. Ahmed, T. et al. (2018) Boosting the Photoluminescence of CsPbX3 (X $=\mathrm{Cl}, \mathrm{Br}$, I) Perovskite Nanocrystals Covering a Wide Wavelength Range by Postsynthetic Treatment with Tetrafluoroborate Salts. Chemistry of Materials 30 (11), 3633-3637

45. Almeida, G. et al. (2018) Role of Acid-Base Equilibria in the Size, Shape, and Phase Control of Cesium Lead Bromide Nanocrystals. ACS Nano 12 (2), 1704-1711 
46. De Roo, J. et al. (2016) Highly Dynamic Ligand Binding and Light Absorption Coefficient of Cesium Lead Bromide Perovskite Nanocrystals. ACS Nano 10 (2), 2071-2081

47. Grisorio, R. et al. (2020) Insights into the role of the lead/surfactant ratio in the formation and passivation of cesium lead bromide perovskite nanocrystals. Nanoscale 12 (2), 623-637

48. Chen, K. et al. (2019) Short-Chain Ligand-Passivated Stable $\alpha$-CsPbl3 Quantum Dot for AllInorganic Perovskite Solar Cells. Advanced Functional Materials 29 (24), 1900991

49. Masi, S. et al. (2020) Stabilization of Black Perovskite Phase in FAPbI3 and CsPbI3. ACS Energy Letters 5 (6), 1974-1985

50. Wang, L. et al. (2017) Scalable Ligand-Mediated Transport Synthesis of Organic-Inorganic Hybrid Perovskite Nanocrystals with Resolved Electronic Structure and Ultrafast Dynamics. ACS Nano 11 (3), 2689-2696

51. Zhang, Y. et al. (2020) A "Tips and Tricks" Practical Guide to the Synthesis of Metal Halide Perovskite Nanocrystals. Chemistry of Materials 32 (13), 5410-5423

52. Swarnkar, A. et al. (2016) Quantum dot-induced phase stabilization of -CsPbl3 perovskite for high-efficiency photovoltaics. Science 354 (6308), 92-95

53. Bodnarchuk, M. I. et al. (2018) Rationalizing and Controlling the Surface Structure and Electronic Passivation of Cesium Lead Halide Nanocrystals. ACS Energy Letters 4 (1), 63-74

54. Liu, J. et al. (2019) Light-Induced Self-Assembly of Cubic CsPbBr3 Perovskite Nanocrystals into Nanowires. Chemistry of Materials 31 (17), 6642-6649

55. Chiba, T. et al. (2017) High-Efficiency Perovskite Quantum-Dot Light-Emitting Devices by Effective Washing Process and Interfacial Energy Level Alignment. ACS Applied Materials \& Interfaces 9 (21), 18054-18060

56. Li, X. et al. (2016) CsPbX3 Quantum Dots for Lighting and Displays: Room-Temperature Synthesis, Photoluminescence Superiorities, Underlying Origins and White Light-Emitting Diodes. Advanced Functional Materials 26 (15), 2435-2445

57. Di Stasio, F. et al. (2018) High-Efficiency Light-Emitting Diodes Based on Formamidinium Lead Bromide Nanocrystals and Solution Processed Transport Layers. Chemistry of Materials 30 (18), 6231-6235

58. Di Stasio, F. et al. (2017) Near-Unity Photoluminescence Quantum Yield in CsPbBr3 Nanocrystal Solid-State Films via Postsynthesis Treatment with Lead Bromide. Chemistry of Materials 29 (18), 7663-7667

59. Wu, H. et al. (2018) Surface ligand modification of cesium lead bromide nanocrystals for improved light-emitting performance. Nanoscale 10 (9), 4173-4178

60. Shynkarenko, Y. et al. (2019) Direct Synthesis of Quaternary Alkylammonium-Capped Perovskite Nanocrystals for Efficient Blue and Green Light-Emitting Diodes. ACS Energy Letters 4 (11), 2703-2711

61. Huang, Y. et al. (2020) DDAB-assisted synthesis of iodine-rich CsPbI3 perovskite nanocrystals with improved stability in multiple environments. Journal of Materials Chemistry C 8 (7), 23812387

62. Zheng, W. et al. (2019) Stabilizing perovskite nanocrystals by controlling protective surface ligands density. Nano Research 12 (6), 1461-1465

63. Song, J. et al. (2018) Room-Temperature Triple-Ligand Surface Engineering Synergistically Boosts Ink Stability, Recombination Dynamics, and Charge Injection toward EQE-11.6\% Perovskite QLEDs. Advanced Materials 30 (30), 1800764

64. Krieg, F. et al. (2018) Colloidal CsPbX3 $(X=\mathrm{Cl}, \mathrm{Br}$, I) Nanocrystals 2.0: Zwitterionic Capping Ligands for Improved Durability and Stability. ACS Energy Letters 3 (3), 641-646

65. Krieg, F. et al. (2019) Stable Ultraconcentrated and Ultradilute Colloids of CsPbX3 $(X=\mathrm{Cl}, \mathrm{Br})$ Nanocrystals Using Natural Lecithin as a Capping Ligand. Journal of the American Chemical Society 141 (50), 19839-19849

66. Pan, J. et al. (2017) Bidentate Ligand-Passivated CsPbI3 Perovskite Nanocrystals for Stable Near-Unity Photoluminescence Quantum Yield and Efficient Red Light-Emitting Diodes. Journal of the American Chemical Society 140 (2), 562-565 
67. Park, S. et al. (2019) Correlation of near-unity quantum yields with photogenerated excitons in X-type ligand passivated CsPbBr3 perovskite quantum dots. Nanoscale Advances 1 (8), 28282834

68. Nenon, D. P. et al. (2018) Design Principles for Trap-Free CsPbX3 Nanocrystals: Enumerating and Eliminating Surface Halide Vacancies with Softer Lewis Bases. Journal of the American Chemical Society 140 (50), 17760-17772

69. Wang, H. et al. (2018) Emission Recovery and Stability Enhancement of Inorganic Perovskite Quantum Dots. The Journal of Physical Chemistry Letters 9 (15), 4166-4173

70. Liu, F. et al. (2017) Highly Luminescent Phase-Stable CsPbI3 Perovskite Quantum Dots Achieving Near 100\% Absolute Photoluminescence Quantum Yield. ACS Nano 11 (10), 1037310383

71. Koscher, B. A. et al. (2017) Essentially Trap-Free CsPbBr3 Colloidal Nanocrystals by Postsynthetic Thiocyanate Surface Treatment. Journal of the American Chemical Society 139 (19), 6566-6569

72. Lu, M. et al. (2019) Ammonium Thiocyanate-Passivated CsPbl3 Perovskite Nanocrystals for Efficient Red Light-Emitting Diodes. The Journal of Physical Chemistry C 123 (37), 22787-22792

73. Huang, G. et al. (2017) Postsynthetic Doping of $\mathrm{MnCl} 2$ Molecules into Preformed CsPbBr3 Perovskite Nanocrystals via a Halide Exchange-Driven Cation Exchange. Advanced Materials 29 (29), 1700095

74. Lu, C.-H. et al. (2020) Doping and ion substitution in colloidal metal halide perovskite nanocrystals. Chemical Society Reviews 49 (14), 4953-5007

75. Begum, R. et al. (2016) Engineering Interfacial Charge Transfer in CsPbBr3 Perovskite Nanocrystals by Heterovalent Doping. Journal of the American Chemical Society 139 (2), 731737

76. Dong, Y. et al. (2018) Precise Control of Quantum Confinement in Cesium Lead Halide Perovskite Quantum Dots via Thermodynamic Equilibrium. Nano Letters 18 (6), 3716-3722

77. Mondal, N. et al. (2018) Achieving Near-Unity Photoluminescence Efficiency for Blue-VioletEmitting Perovskite Nanocrystals. ACS Energy Letters 4 (1), 32-39

78. Mir, W. J. et al. (2020) Lanthanide doping in metal halide perovskite nanocrystals: spectral shifting, quantum cutting and optoelectronic applications. NPG Asia Materials 12 (1),

79. Kroupa, D. M. et al. (2018) Quantum-Cutting Ytterbium-Doped CsPb(Cl1-xBrx)3 Perovskite Thin Films with Photoluminescence Quantum Yields over 190\%. ACS Energy Letters 3 (10), 23902395

80. Alam, F. et al. (2019) Eu2+: A suitable substituent for Pb2+ in CsPbX3 perovskite nanocrystals? The Journal of Chemical Physics 151 (23), 231101

81. Ji, S. et al. (2020) Near-Unity Red Mn2+ Photoluminescence Quantum Yield of Doped $\mathrm{CsPbCl} 3$ Nanocrystals with Cd Incorporation. The Journal of Physical Chemistry Letters 11 (6), 2142-2149

82. Parobek, D. et al. (2016) Exciton-to-Dopant Energy Transfer in Mn-Doped Cesium Lead Halide Perovskite Nanocrystals. Nano Letters 16 (12), 7376-7380

83. Yong, Z.-J. et al. (2018) Doping-Enhanced Short-Range Order of Perovskite Nanocrystals for Near-Unity Violet Luminescence Quantum Yield. Journal of the American Chemical Society 140 (31), 9942-9951

84. Cai, T. et al. (2018) Synthesis of All-Inorganic Cd-Doped CsPbCl3 Perovskite Nanocrystals with Dual-Wavelength Emission. The Journal of Physical Chemistry Letters 9 (24), 7079-7084

85. Milstein, T. J. et al. (2018) Picosecond Quantum Cutting Generates Photoluminescence Quantum Yields Over $100 \%$ in Ytterbium-Doped CsPbCl3 Nanocrystals. Nano Letters 18 (6), 3792-3799

86. Cai, T. et al. (2020) Mn2+/Yb3+Codoped CsPbCl3 Perovskite Nanocrystals with TripleWavelength Emission for Luminescent Solar Concentrators. Advanced Science, 2001317 
87. Gualdrón-Reyes, A. F. et al. (2021) Engineering Sr-doping for enabling long-term stable FAPb1-xSrxl3 quantum dots with $100 \%$ photoluminescence quantum yield. Journal of Materials Chemistry C 9 (5), 1555-1566

88. Brown, A. A. M. et al. (2020) Lead Halide Perovskite Nanocrystals: Room Temperature Syntheses toward Commercial Viability. Advanced Energy Materials, 2001349

89. Deng, W. et al. (2018) Organic-inorganic hybrid perovskite quantum dots for light-emitting diodes. Journal of Materials Chemistry C 6 (18), 4831-4841

90. Gonzalez-Carrero, S. et al. (2016) The Luminescence of CH3NH3PbBr3Perovskite Nanoparticles Crests the Summit and Their Photostability under Wet Conditions is Enhanced. Small 12 (38), 5245-5250

91. Dai, S.-W. et al. (2018) Perovskite Quantum Dots with Near Unity Solution and Neat-Film Photoluminescent Quantum Yield by Novel Spray Synthesis. Advanced Materials 30 (7), 1705532 92. Veldhuis, S. A. et al. (2017) Benzyl Alcohol-Treated CH3NH3PbBr3Nanocrystals Exhibiting High Luminescence, Stability, and Ultralow Amplified Spontaneous Emission Thresholds. Nano Letters 17 (12), 7424-7432

93. Jancik Prochazkova, A. et al. (2019) Proteinogenic Amino Acid Assisted Preparation of Highly Luminescent Hybrid Perovskite Nanoparticles. ACS Applied Nano Materials 2 (7), 4267-4274

94. Paul, S.; Samanta, A. (2019) N-Bromosuccinimide as Bromide Precursor for Direct Synthesis of Stable and Highly Luminescent Green-Emitting Perovskite Nanocrystals. ACS Energy Letters 5 (1), 64-69

95. Imran, M. et al. (2018) Benzoyl Halides as Alternative Precursors for the Colloidal Synthesis of Lead-Based Halide Perovskite Nanocrystals. Journal of the American Chemical Society 140 (7), 2656-2664

96. Sun, C. et al. (2018) A new method to discover the reaction mechanism of perovskite nanocrystals. Dalton Transactions 47 (45), 16218-16224

97. Dutta, A. et al. (2019) Near-Unity Photoluminescence Quantum Efficiency for All CsPbX3 $(\mathrm{X}=\mathrm{Cl}, \mathrm{Br}$, and I) Perovskite Nanocrystals: A Generic Synthesis Approach. Angewandte Chemie International Edition 58 (17), 5552-5556

98. Dutta, A. et al. (2018) Tuning the Size of CsPbBr3 Nanocrystals: All at One Constant Temperature. ACS Energy Letters 3 (2), 329-334

99. Chen, T. et al. (2020) Ionic liquid assisted preparation and modulation of the photoluminescence kinetics for highly efficient CsPbX3 nanocrystals with improved stability. Nanoscale 12 (17), 9569-9580

100. Grisorio, R. et al. (2020) A new route for the shape differentiation of cesium lead bromide perovskite nanocrystals with near-unity photoluminescence quantum yield. Nanoscale 12 (32), 17053-17063 


\section{Figures}

(A)

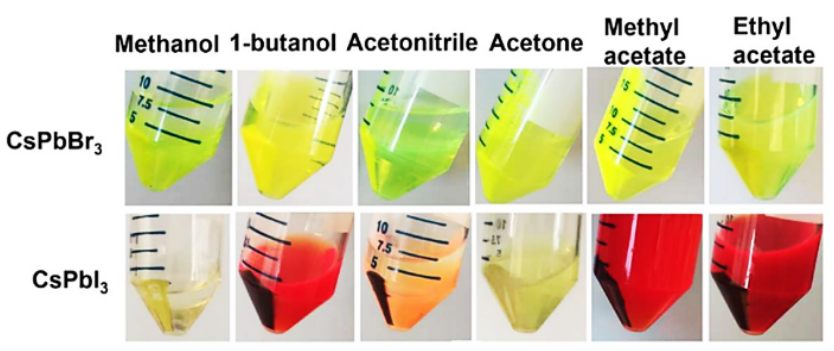

(B)

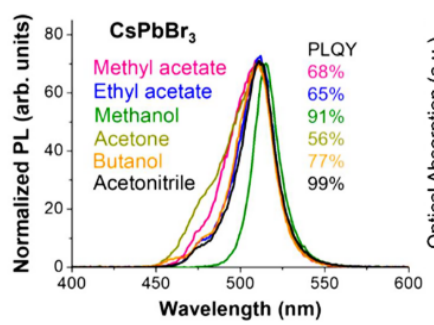

(C)

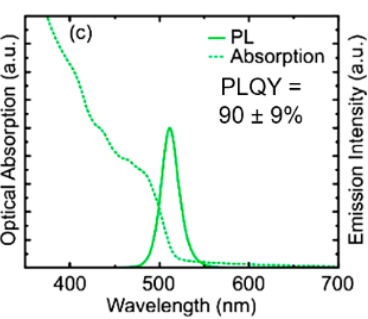

(D)

Figure 1. Purification of perovskite nanocrystals with antisolvents. (A) Photographs of $\mathrm{CsPbr}_{3}$ and $\mathrm{CsPbI}_{3}$ nanocrystals dispersed in antisolvents with different polarity (Polarity increases from ethyl acetate $\rightarrow$ methanol). (B) PL spectra and PLQY of $\mathrm{CsPBr}_{3}$ nanocrystals dispersed in hexane, purified with high-polar and low-polar antisolvents. Reproduced with permission from [51]. (C) Optical absorption, PL, PLQY and (D) time-resolved PL measurements of $\mathrm{FAPbBr}_{3}$ nanocrystals dispersed in toluene before and after purification $\mathrm{OA}+\mathrm{PbBr}_{2}+\mathrm{MeOAc}$. Reproduced with permission from [57].

(A)
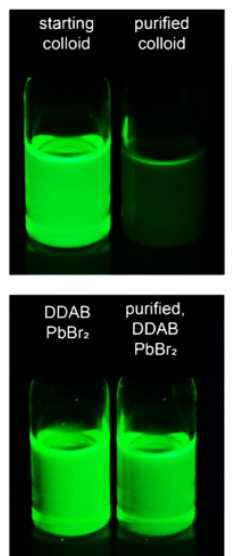

(B)

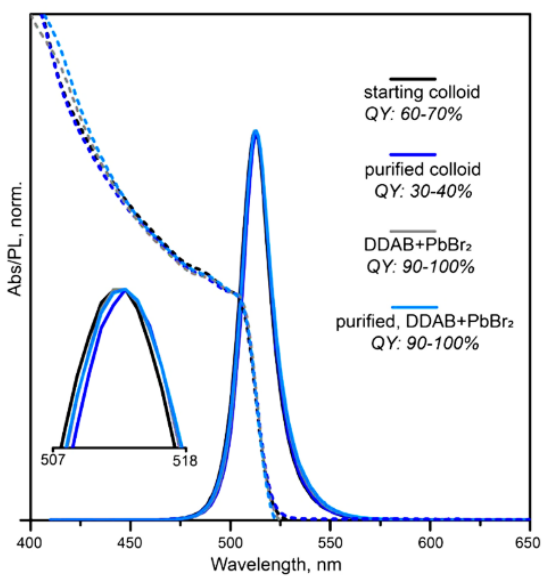

(C)

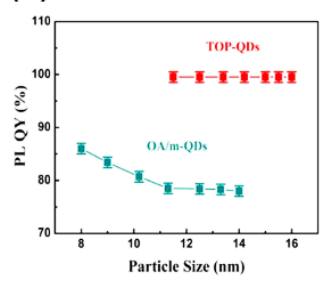

(D)

(E)

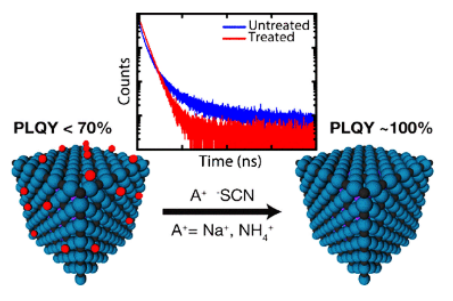

Figure 2. Repairing of the perovskite nanocrystals surface. (A) photographs of the greenluminescent colloidal solutions of $\mathrm{CsPbr}_{3}$ nanocrystals and their corresponding (B) PLQY, absorption and PL spectra before and after purification stage with acetone, in presence and absence of $\mathrm{DDAB}$ or $\mathrm{DDAB}+\mathrm{PbBr}_{2}$ after the post-synthetic treatment. Reproduced with permission from [53]. (C) PLQY of $\mathrm{CsPbI}_{3}$ nanocrystals with and without adding TOP during their synthesis, by varying the particle size. (D) PLQY as a function of the storage time for 
standard and TOP-modified $\mathrm{CsPbI}_{3}$ nanocrystals. Reproduced with permission from [70].

Typical PL decay and schematic representation of the surface defects reparation in $\mathrm{CsPbr}_{3}$ nanocrystals by using $\mathrm{NaSCN}$ and $\mathrm{NH}_{4} \mathrm{SCN}$. Reproduced with permission from [71].

(A)

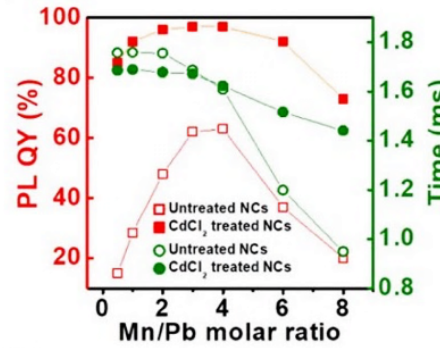

(C)

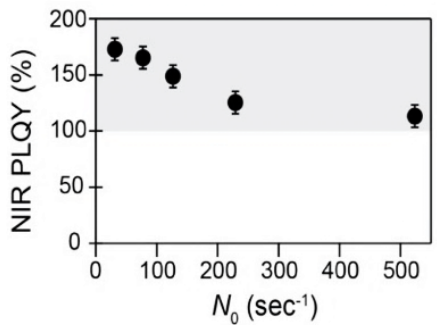

(B)

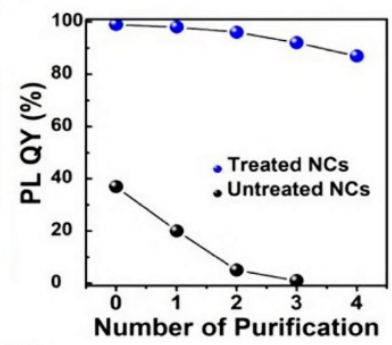

(D)

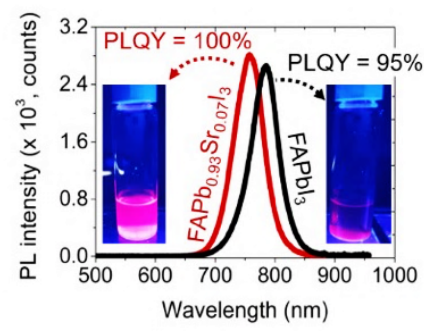

Figure 3. Partial substitution of $\mathrm{Pb}$ into perovskite nanocrystals. (A) PLQY and PL lifetime of the $\mathrm{Mn}^{2+}: \mathrm{CsPCl}_{3}$ nanocrystals by varying the amount of $\mathrm{Mn}^{2+}$, with and without $\mathrm{CdCl}_{2}$ treatment. (B) PLQY of $\mathrm{Mn}^{2+}: \mathrm{CsPCCl}_{3}$ nanocrystals $(\mathrm{Mn}: \mathrm{Pb}=6: 1)$ in function of the number of purifications with acetone before and after Cd incorporation. Reproduced with permission from [81]. (C) NIR PLQY of the $5.2 \% \mathrm{Yb}^{2+}$-doped $\mathrm{CsPbCl}_{3}$ nanocrystals as a function of the photoexcitation rate, carried out by varying the intensity of the excitation source $(\lambda=380 \mathrm{~nm})$. Reproduced with the permission from [85]. (D) PL spectra and calculated PLQY of $\mathrm{FAPbI}_{3}$ and $\mathrm{FAPb}_{0.93} \mathrm{Sr}_{0.07} \mathrm{I}_{3}$ nanocrystals and their corresponding photographies under UV light (inset).

(A)

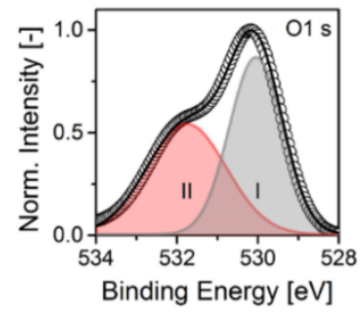

(D)

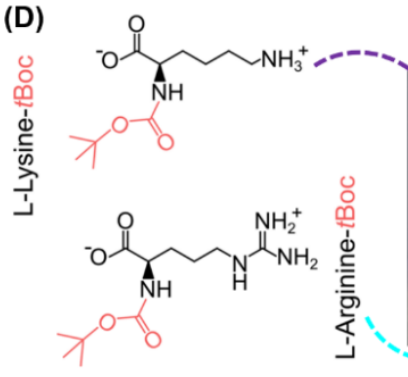

(B)
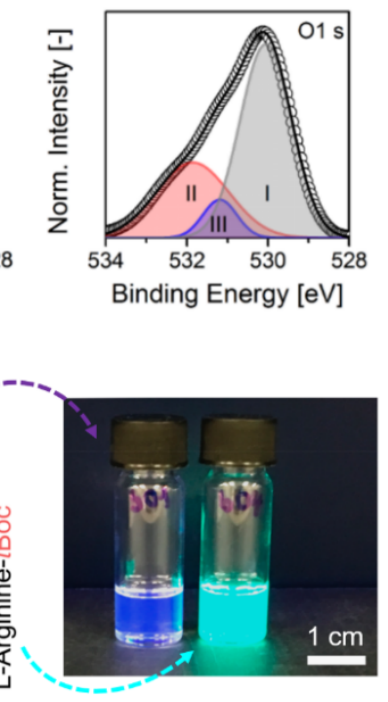

(C)

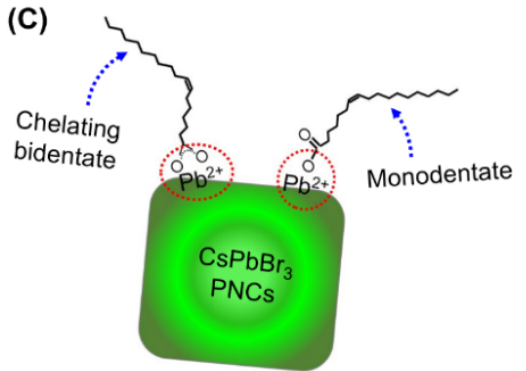

(E)

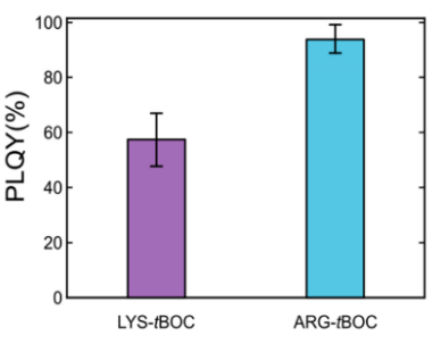


Figure 4. Characterization of high-quality perovskite nanocrystals synthesized by modifying the synthetic route based on ligand-assisted reprecipitation method (LARP). (A,B) High-resolution XPS O 1s spectra of $\mathrm{MAPbBr}_{3}$ perovskite nanocrystals synthesized by LARP in presence and absence of benzyl alcohol, respectively. Regions I and III are associated to the bidentate and monodentate binding modes of the carboxylic acid (OA), while region II corresponds to the presence of $\mathrm{O}-\mathrm{H}$ groups from water during perovskite synthesis. (C) Representative scheme of the bidentate and monodentate profits from carboxylate ligand bounded to the nanocrystal surface. Reproduced and adapted with the permission from [92]. (D) Photographs of the $\mathrm{MAPbBr}_{3}$ perovskite nanocrystals synthesized by using L-Lysine-tBoc (LYS-tBoc) and L-Arginie-tBoc (ARG-tBoc) as capping ligands through LARP. (E) Calculated PLQY from the LYS-tBoc- and ARG-tBoc-capped $\mathrm{MAPbBr}_{3}$ samples. Reproduced and adapted with the permission from [93].

(A)

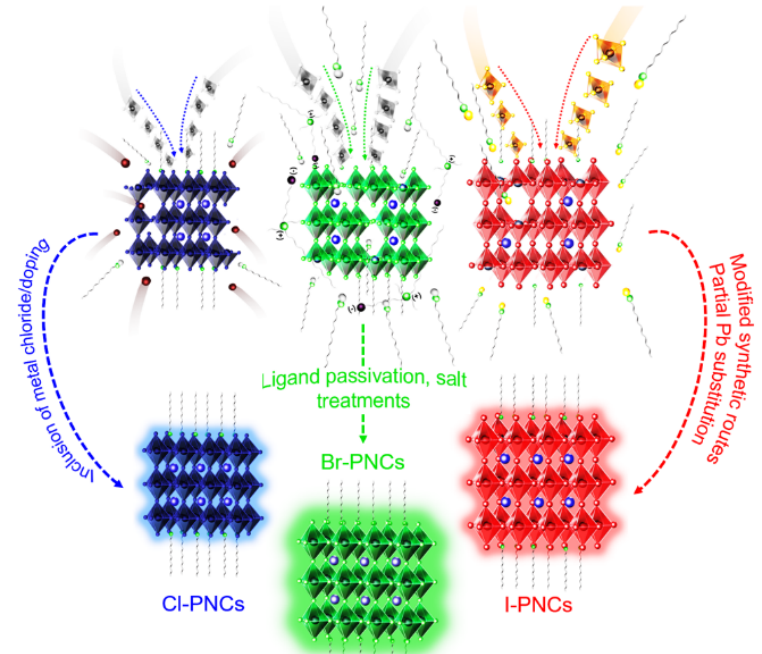

(B)

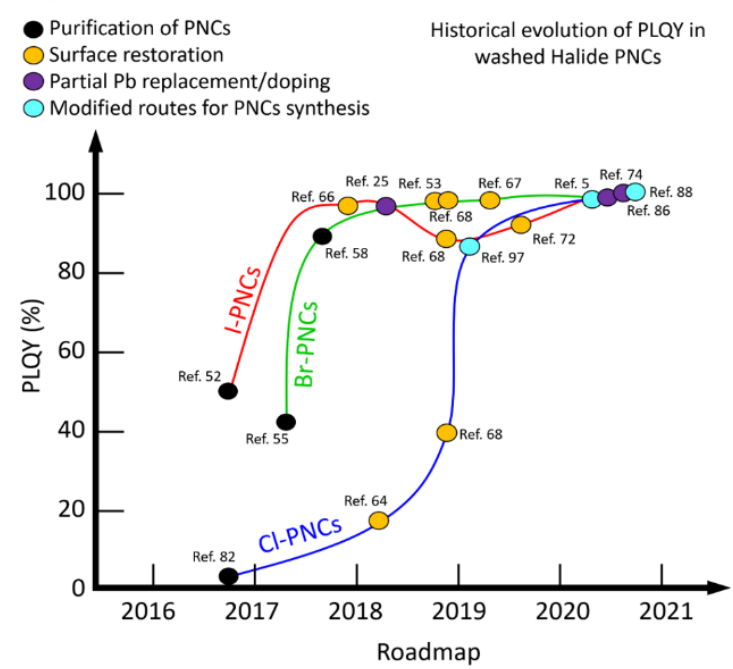

Figure 5. a) Schematic representation of the main alternatives for achieving high-quality perovskite nanocrystals with near-unity photoluminescence quantum yield, depending on the type of halide. Approaches were chosen based on the effectiveness of reduction of halide vacancies and restoration of surface defects. b) Evolution of PLQY of washed PNCs according to the different methodologies. 